Letter to the Editor

\title{
Analysis of the effects of resistance training on circadan rhythm of endocrine hormones
}

\author{
Ardalan Shariat ${ }^{1}$, Ina Shaw ${ }^{2,3}$, Brandon S. Shaw ${ }^{3}$, Hassan Sadeghi ${ }^{1}$ \\ ${ }^{1}$ University Putra Malaysia, Serdang, Malaysia \\ ${ }^{2}$ Monash University (South Africa Campus), Ruimsig, Republic of South Africa \\ ${ }^{3}$ University of Johannesburg, Johannesburg, Republic of South Africa
}

Received 25 April 2015, Accepted 7 May 2015

(C) 2015, Shariat A., Shaw I., Shaw B.S., Sadeghi H.

(C) 2015, Russian Open Medical Journal

Abstract: In the past decade, the number of the studies conducted on the effects of resistance training on circadian rhythm of endocrine hormones is increasing exponentially. The aim of this report is to explore the current and prevailing studies in an attempt to elucidate the findings of resistance training on circadian rhythm of endocrine hormones. Analysis of the available research from 2001 until 2015 demonstrated a wide variety of methodologies and sampled populations. However, all sampled studies made use of a pre-test, post-test experimental design taking place during the day. In addition, the primary hormones assessed were testosterone and cortisol, sampled via saliva. Collectively the sampled studies demonstrated that resistance training is capable of affecting the circadian rhythm of endocrine hormone secretion. However, it must be noted that these alterations were primary following training and the alterations transient in nature, with endocrine responses returning to normal circadian rhythm ranges via natural feedback mechanisms.

Keywords: cortisol, hormones resistance exercise, testosterone, weight training

Cite as Shariat A, Shaw I, Shaw BS, Sadeghi H. Analysis of the effects of resistance training on circadan rhythm of endocrine hormones. Russian Open Medical Journal 2015; 4: e0302.

Correspondence to Ardalan Shariat. Phone: +60173365494. Email: ardalansh2002@gmail.com

Exercise training is known to induce a hormonal adaptation process. However, any time-of-day effect of exercise on hormonal responses remains largely unknown. This is especially true for resistance training in that optimal adaptations to resistance training also seem to occur in the late afternoon, which is interesting, since hormone (cortisol and, particularly, testosterone) concentrations are higher in the morning. In the past decade, several studies have attempted to determine, if resistance training was capable of altering these time-of-day or circadian rhythm effects of hormones. Specifically, W. Kraemer et al. [1] measured the effect of intensive resistance exercise on the circadian rhythm of salivary testosterone and cortisol among recreational bodybuilders. The results of the study demonstrated that intensive resistance training can affect the secretion of testosterone and cortisol. However, these changes were only evident following training and lasted for approximately one hour, at which point concentrations of both hormones returned to resting or pre-exercise levels. These findings were supported by $A$. Shariat et al. [2] who measured the effect of heavy resistance exercise on the circadian rhythm of cortisol among young bodybuilders. Problematically, both of these studies failed to make use of a comparative control group.

A later study [3] attempted to compare the effect of soy and whey protein supplements on acute hormonal responses following resistance training. Using a balanced placebo design, results of this study indicated that whey protein can acutely affect testosterone concentrations following resistance training while soy supplementation following resistance training does not. However, these supplements did not affect the circadian rhythm of these hormones.

In an attempt to more definitively assess hormonal responses to resistance training on various athletic populations, A. Shariat et al. [4] made use of a balanced crossover research design to rest and training days with regard to hormonal response to resistance training. Using a sample of fitness trainees, it was hypothesized that the lower muscle mass of these subjects, when compared to previous studies making use of bodybuilders, would yield differences in hormonal responses to the resistance training. However, the results of this study again indicated that resistance training only acutely affects cortisol, with no long-term circadian rhythm alterations. Similarly, J. Ghigiarelli et al. [5] examined the effect of multi-joint, large muscle group, heavy resistance training on athletes' testosterone concentrations. Again, this study demonstrated increased testosterone concentration immediately following resistance training, without long-term alterations.

In an attempt to determine and compare the effects of different types of resistance training on the circadian rhythm of endocrine hormones, a cross-sectional study was employed on Judoists [6]. This study confirmed previous findings when it found that resistance training, irrespective of the type, failed to alter the circadian rhythm of hormone concentrations. Most recently, in 2015, Shariat et al. [7] again made use of bodybuilders in a longitudinal study in an attempt to determine the effect of resistance training on hormone concentrations. This study made 
use of a control group, which allowed for comparisons within-and between-groups comparisons at pre-test and post-test. Although this study made use of a much improved methodological design when compared to previous studies, the results of this study once again demonstrated acute changes in hormonal concentrations without alterations in the long-term.

\section{Conclusion}

In conclusion, the available data suggests that there is a strong link between resistance training and hormonal responses. However, research also indicates that this effect is transient and different types of resistance training cannot change the circadian and diurnal rhythm of cortisol and/or testosterone in varying populations of athletes. These findings have significant implications for athletes attempting to improve training and competition performance. This is so since athletes, their conditioning specialists and coaches attempt to chronically increase concentrations of the anabolic hormones, such as testosterone and growth hormone, while simultaneously trying to decrease the catabolic hormones, such as cortisol [7, 8]. The findings of previous research suggest that other internal factors, such as hypothalamus, control this rhythm of hormone concentrations, however, more research is needed to determine the precise mechanism responsible for transient, but not chronic hormonal responses to resistance training. To date, research has indicated that the use of ergogenic aids, such as anabolic steroids is the most effective means of positively adapting the long-term effects of selected hormones, however, the side-effects associated with the use of such ergogenic aids far outweighs their use [3].

\section{Conflicts of Interest}

The authors declare that they have no financial, consultant, institutional and other relationships that might lead to bias or a conflict of interest.

\section{References}

1. Fry AC, Kraemer WJ, Stone MH, Perry Koziris L, Thrush JT, Fleck SJ. Relationships between serum testosterone, cortisol, and weightlifting performance. J Strength Cond Res 2000; 14(3): 338-342.

2. Shariat A, Kargarfard M, Sharifi GR. The effect of heavy resistance exercise on circadian rhythm of salivary cortisol in male body building athletes. J ISFAHAN Med Sch 2012; 29: 2400-2412.

3. Kraemer WJ, Solomon-Hill G, Volk BM, Kupchak BR, Looney DP, DunnLewis $C$, et al. The effects of soy and whey protein supplementation on acute hormonal responses to resistance exercise in men. J Am Coll Nutr 2013; 32(1): 66-74. (doi: 10.1080/07315724.2013.770648) (PMID: 24015701)

4. Shariat A, Kargarfard M, Nekooei P, Majlesi S. Comparison between salivary cortisol release in rest and training days in male fitness trainee. Int J Kinesiol Sport Sci 2013; 1: 9-15.

5. Ghigiarelli JJ, Sell KM, Raddock JM, Taveras K. Effects of strongman training on salivary testosterone levels in a sample of trained men. $J$ Strength Cond Res 2013; 27(3): 738-747. (PMID: 23443220) (doi: 10.1519/JSC.0b013e3182578115)

6. Shariat A, Kargarfard M, Mohd Tamrin SB, Danaee M, Karimi H. Strength-training and biological rhythm of male sex hormone among judoists. Biol Rhythm Res 2014; 45: 1-14. (doi: 10.1080/09291016.2014.884304)

7. Shariat A, Kargarfard M, Danaee M, Tamrin SBM. Intensive resistance exercise and circadian salivary testosterone concentrations among young male recreational lifters. J Strength Cond Res 2015; 29(1): 151158. (doi: 10.1519/JSC.0000000000000632) (PMID: 25051005)

8. Shaw BS, Shaw I, Brown GA. Comparison of resistance and concurrent resistance and endurance training regimes in the development of strength. J Strength Cond Res 2009; 23(9): 2507-2514. (doi: 10.1519/JSC.0b013e3181bc191e)

\section{Authors:}

Ardalan Shariat - PhD candidate, Specialist, Department of Occupational Health, Faculty of Medicine and Health Sciences, University Putra Malaysia, Serdang, Malaysia.

Ina Shaw - PhD, Research Manager and Visiting Professor, Research and Development, Monash University (South Africa Campus), Ruimsig, Republic of South Africa; Department of Sport and Movement Studies, University of Johannesburg, Doornfontein, Johannesburg, 2028, Republic of South Africa.

Brandon S. Shaw - PhD, Full Professor, Department of Sport and Movement Studies, University of Johannesburg, Doornfontein, Johannesburg, 2028, Republic of South Africa.

Hassan Sadeghi - PhD candidate, Specialist, Department of Sport Studies, University Putra Malaysia, Serdang, Malaysia. 